\title{
Is the Universe As Large As It Can Be?*
}

\author{
John Byron Manchak
}

[Forthcoming in Erkenntnis.]

\begin{abstract}
In this note, we cast doubt on the requirement of spacetime inextendibility; it is not at all clear that our universe is "as large as it can be."
\end{abstract}

\section{Introduction}

A number of experts in general relativity seem to be committed to the following (Earman 1995, 32):

"Metaphysical considerations suggest that to be a serious candidate for describing actuality, a spacetime should be [inextendible]. For example, for the Creative Force to actualize a proper subpart of a larger spacetime would seem to be a violation of Leibniz's principles of sufficient reason and plenitude. If one adopts the image of spacetime as being generated or built up as time passes then the dynamical version of the principle of sufficient reason would ask why the Creative Force would stop building if it is possible to continue."

An important mathematical theorem due to Geroch (1970) underpins such metaphysical views: Every extendible model of general relativistic spacetime has an inextendible extension. Indeed, the theorem is foundational for those who "regard inextendibility as a reasonable physical condition to be imposed on models of the universe" (Geroch 1970, 262). In this note, we cast doubt on the requirement of spacetime inextendibility. ${ }^{1}$ We construct a model of the universe with a "physically reasonable" property which cannot be extended with the property to be inextendible. In other words, it seems that when attention is restricted to a more reasonable subset of models of general relativity, there is no analogue to the Geroch (1970) theorem.

*Thanks go to Jeff Barrett, Thomas Barrett, Bob Geroch, David Malament, Jim Weatherall, and two anonymous referees for helping to improve an earlier draft.

${ }^{1}$ For two additional arguments against the requirement of spacetime inextendibility, see Manchak (2011, 2016). 


\section{Preliminaries}

We begin with a few preliminaries concerning the relevant background formalism of general relativity (Wald 1984). An $n$-dimensional, relativistic spacetime (for $n \geq 2$ ) is a pair of mathematical objects $\left(M, g_{a b}\right)$. $M$ is a connected $n$-dimensional Hausdorff manifold (without boundary) that is smooth (infinitely differentiable). Here, $g_{a b}$ is a smooth, non-degenerate, pseudo-Riemannian metric of Lorentz signature $(+,-, \ldots,-)$ defined on $M$. We say two spacetimes $\left(M, g_{a b}\right)$ and $\left(M^{\prime}, g_{a b}^{\prime}\right)$ are isometric if there is a diffeomorphism $\varphi: M \rightarrow M^{\prime}$ such that $\varphi_{*}\left(g_{a b}\right)=g_{a b}^{\prime}$.

A spacetime $\left(M, g_{a b}\right)$ is extendible if there exists a spacetime $(M, g)$ and an isometric embedding $\varphi: M \rightarrow M^{\prime}$ such that $\varphi[M]$ is a proper subset of $M^{\prime}$. Here, the spacetime $\left(M^{\prime}, g_{a b}^{\prime}\right)$ is an extension of $\left(M, g_{a b}\right)$. A spacetime is inextendible if it has no extension. A $\mathscr{P}$-spacetime is a spacetime with property $\mathscr{P}$. A $\mathscr{P}$-spacetime $\left(M^{\prime}, g_{a b}^{\prime}\right)$ is a $\mathscr{P}$-extension of a $\mathscr{P}$-spacetime $\left(M, g_{a b}\right)$ if $\left(M^{\prime}, g_{a b}^{\prime}\right)$ is an extension of $\left(M, g_{a b}\right)$. A $\mathscr{P}$ spacetime is $\mathscr{P}$-extendible if it has a $\mathscr{P}$-extension and is $\mathscr{P}$-inextendible otherwise.

For each point $p \in M$, the metric assigns a cone structure to the tangent space $M_{p}$. Any tangent vector $\xi^{a}$ in $M_{p}$ will be timelike if $g_{a b} \xi^{a} \xi^{b}>0$, null if $g_{a b} \xi^{a} \xi^{b}=0$, or spacelike if $g_{a b} \xi^{a} \xi^{b}<0$. Null vectors create the cone structure; timelike vectors are inside the cone while spacelike vectors are outside. A time orientable spacetime is one that has a continuous timelike vector field on $M$. A time orientable spacetime allows one to distinguish between the future and past lobes of the light cone. In what follows, it is assumed that spacetimes are time orientable.

For some open (connected) interval $I \subseteq \mathbb{R}$, a smooth curve $\gamma: I \rightarrow$ $M$ is timelike if the tangent vector $\xi^{a}$ at each point in $\gamma[I]$ is timelike. Similarly, a curve is null (respectively, spacelike) if its tangent vector at each point is null (respectively, spacelike). A curve is causal if its tangent vector at each point is either null or timelike. A causal curve is future directed if its tangent vector at each point falls in or on the future lobe of the light cone.

An extension of a curve $\gamma: I \rightarrow M$ is a curve $\gamma^{\prime}: I^{\prime} \rightarrow M$ such that $I$ is a proper subset of $I^{\prime}$ and $\gamma(s)=\gamma^{\prime}(s)$ for all $s \in I$. A curve is maximal if it has no extension. A curve $\gamma: I \rightarrow M$ in a spacetime $\left(M, g_{a b}\right)$ a geodesic if $\xi^{a} \nabla_{a} \xi^{b}=\mathbf{0}$ where $\xi^{a}$ is the tangent vector and $\nabla_{a}$ is the unique derivative operator compatible with $g_{a b}$. A point $p \in M$ is a future endpoint of a future directed causal curve $\gamma: I \rightarrow M$ if, for every neighborhood $O$ of $p$, there exists a point $t_{0} \in I$ such that $\gamma(t) \in O$ for all $t>t_{0}$. A past endpoint is defined similarly. A causal curve is inextendible if it has no future or past endpoint. For any set $S \subseteq M$, we define the domain of dependence of $S$, written $D(S)$, to be the set of points $p \in M$ such that every causal inextendible causal curve through $p$ intersects $S$. A causal geodesic $\gamma: I \rightarrow M$ in a spacetime $(M, g)$ is past incomplete if it is maximal and there is an $r \in \mathbb{R}$ such that $r<s$ for all $s \in I$. 


\section{Example}

As mentioned above, Geroch (1970) showed that every extendible spacetime has an inextendible extension. Now, the question examined here is this: Is it the case that for all "physically reasonable" properties $\mathscr{P}$, every $\mathscr{P}$-extendible $\mathscr{P}$-spacetime has a $\mathscr{P}$-inextendible $\mathscr{P}$-extension? No.

Of course, spacetime properties may be considered "physically reasonable" in various senses. In what follows, let us restrict attention to those properties usually taken to be satisfied by (models of) our own universe. We will say that a spacetime has property $\mathscr{P}$ if every inextendible timelike geodesic in the spacetime is past incomplete. The "big bang" in our own universe presumably renders $\mathscr{P}$ "physically reasonable" in our sense.

Example. Let $\left(\mathbb{R}^{2}, \eta\right)$ be two-dimensional Minkowski spacetime in standard $(t, x)$ coordinates. For each $i \in \mathbb{Z}^{+}$let $S_{i}$ be the (disjoint) set $\left\{(t, x) \in \mathbb{R}^{2}: t=-i\right.$ and either $x \leq-1 / i$ or $\left.1 / i \leq x\right\}$. Let $S=\cup\left\{S_{i}\right\}$. Consider a smooth positive function $\Omega: \mathbb{R}^{2}-S \rightarrow \mathbb{R}$ such that (i) $\Omega=1$ for all points in $\mathbb{R}^{2}-D(S)$ and (ii) $\Omega$ approaches zero as $S$ is approached along every timelike curve contained in $D(S)$. Now consider $(M, g)$ where $M=\mathbb{R}^{2}-S$ and $g=\Omega^{2} \eta$. Note that $(M, g)$ is inextendible. Let $\gamma: I \rightarrow$ $M$ be any inextendible timelike geodesic. One can verify that $\gamma$ is past incomplete except in the case where $\gamma[I]$ is the line $x=0$. (Every other inextendible geodesic enters $D(S)$ in the past direction and therefore must approach $S$.) Let $N$ be the set $\left\{(t, x): t \in \mathbb{Z}^{-}\right.$and $\left.x=0\right\}$. Consider the spacetime $(M-N, g)$ (see Figure 1$)$.

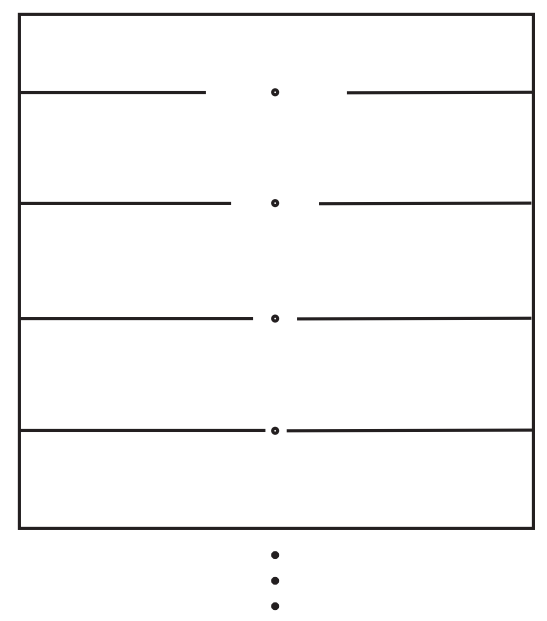

Figure 1: The spacetime $(M-N, g)$.

Because of the infinite number of "missing points" $N$, every inextendible timelike geodesic in $(M-N, g)$ is past incomplete. So $(M-N, g)$ is a $\mathscr{P}$-spacetime. Note that any extension to $(M-N, g)$ is, for some nonempty set $P \subseteq N$, the spacetime $(M-(N-P), g)$. One can show that 
$(M-(N-P), g)$ is a $\mathscr{P}$-extension of the $\mathscr{P}$-spacetime $(M-N, g)$ if and only if $N-P$ is an infinite set. (If $N-P$ is infinite, there is no "lowest" point in $N-P$. So, every inextendible timelike geodesic whose image is a subset of the line $x=0$ will approach some point in $N-P$ in the past direction. Thus, the geodesic will be past incomplete. On the other hand, if $N-P$ is finite, then there will be a "lowest" point in $N-P$. So, the inextendible timelike geodesic whose image is that portion of the line $x=0$ below the lowest point in $N-P$ will be past complete.) Since there exist infinite sets $N-P$ (take the set $N-\{q\}$ for any $q \in N$ for example), it follows that $(M-N, g)$ is $\mathscr{P}$-extendible. Now, consider any $\mathscr{P}$-extension $(M-(N-P), g)$ of the $\mathscr{P}$-spacetime $(M-N, g)$. So $N-P$ is an infinite set. Let $R$ be such that $P \subset R \subset N$ and $N-R$ is an infinite set. (Such a set must exist. Take the set $P \cup\{q\}$ for any $q \in N-P$ for example.) It follows that $(M-(N-R), g)$ is a $\mathscr{P}$-extension to $(M-(N-P), g)$. So, $(M-(N-P), g)$ is $\mathscr{P}$-extendible. Since $(M-(N-P), g)$ was arbitrarily chosen, we know that the $\mathscr{P}$-extendible $\mathscr{P}$-spacetime $(M-N, g)$ has no $\mathscr{P}$-inextendible $\mathscr{P}$-extension.

\section{Conclusion}

One might protest that the above example is not physically reasonable in some sense. Fine. But the point is "to demonstrate by some example that a certain assertion is false, or that a certain line of argument cannot work" (Geroch and Horowitz 1979, 221). In this case, the assertion demonstrated to be false is: It is clear that the Creative Force can build our universe to be "as large as it can be."

\section{References}

[1] Earman, J. (1995). Bangs, Crunches, Whimpers, and Shrieks: Singularities and Acausalities in Relativistic Spacetimes. Oxford: Oxford University Press.

[2] Geroch, R. (1970), "Singularities", in M. Carmeli, S. I. Fickler, and L. Witten (eds.), Relativity. New York: Plenum Press, 259-291.

[3] Geroch, R., and Horowitz, G. (1979). "Global Structure of Spacetimes," in S. W. Hawking and W. Israel (eds), General Relativity: An Einstein Centenary Survey. Cambridge: Cambridge University Press, 212-293.

[4] Manchak, J. (2011). "What is a Physically Reasonable Spacetime?" Philosophy of Science, 78: 410-420.

[5] Manchak, J. (2016). "Epistemic "Holes" in Spacetime." Forthcoming in Philosophy of Science.

[6] Wald, R. (1984). General Relativity. Chicago: University of Chicago Press. 